\title{
Distributed Coordination of Fractional Dynamical Systems with Exogenous Disturbances
}

\author{
Hongyong Yang, Fujun Han, Fei Liu, Huixia Liu, and Mei Zhao \\ School of Information and Electrical Engineering, Ludong University, Yantai 264025, China \\ Correspondence should be addressed to Hongyong Yang; hongyongyang@yahoo.com
}

Received 13 February 2014; Accepted 18 June 2014; Published 21 July 2014

Academic Editor: Adem Kılıçman

Copyright (C) 2014 Hongyong Yang et al. This is an open access article distributed under the Creative Commons Attribution License, which permits unrestricted use, distribution, and reproduction in any medium, provided the original work is properly cited.

\begin{abstract}
Distributed coordination of fractional multiagent systems with external disturbances is studied. The state observer of fractional dynamical system is presented, and an adaptive pinning controller is designed for a little part of agents in multiagent systems without disturbances. This adaptive pinning controller with the state observer can ensure multiple agents' states reaching an expected reference tracking. Based on disturbance observers, the controllers are composited with the pinning controller and the state observer. By applying the stability theory of fractional order dynamical systems, the distributed coordination of fractional multiagent systems with external disturbances can be reached asymptotically.
\end{abstract}

\section{Introduction}

Distributed coordination of multiagent systems has become an active research field in the systems and controls society because of its importance applications in both civilian and military sectors. A large number of distributed coordination scenarios have been studied, like formation control of the unmanned aerial vehicles, advanced autonomous monitoring system, attitude control of satellites, distributed sensor networks, undersea robots cooperation control, cloud computing systems composed of multiservers, and so forth [1-8].

The study results of the multiagent systems are mainly on the integer-order systems whose dynamical equations are first-order, second-order, or high-order differential equation. However, many multiagent systems cannot be characterized with the integer-order systems in the complex physical environment, while they only are described with fractional order (noninteger) differential equations $[9,10]$. In actual complex environment, it can reveal the nature of the characteristics and behavior for the physical system with fractional-order feature described by fractional dynamics [11, 12]. Recently, fractional calculus has attracted a significant number of researchers from physics and engineering. Many dynamical systems such as dielectric polarization, viscoelastic systems, and electromagnetic waves have turned out to be modeled by fractional-order differential equations, and many important results for fractional-order dynamical systems have been obtained [13-17]. Many multiple agents have emerged out the fractional cooperative behavior, for example: flocking and food searching of colony system by means of the individual secretions, submarine underwater robots in the undersea with a large number of microorganisms and viscous substances, and unmanned aerial vehicles running in the complex space environment [11, 12]. Cao and Ren have studied distribution coordination of multiagent systems based on the fractional-order systems [18] and obtained the relationship between the individuals' number and the fractional order on the stability of multiagent systems. Yang et al. have researched the collaboration control of fractional-order multiagent systems with communication delays [19-21]. Motivated by the broad application of distributed cooperation in multiagent systems and the fact that many practical physical systems are demonstrated with fractional-order dynamics, the distributed coordination of fractional-order networked systems will be studied in this paper. 
In this paper, we intend to study the fractional dynamical systems with external disturbances. In the movement process of multiple agents, various disturbances, such as stochastic noises, modeling errors, external interferences, and communication delays, will influence the performance of multiagent systems. Recently, as an effective robust control way, methods of the disturbance-observer have been widely used in the fields of mechanical, electrical, power, intelligence agent and structural engineering [22-27]. Guo and Chen [22] presented a disturbance observers based control method from the linear system to a dynamic nonlinear model with disturbance for the first time. Wei et al. [23] studied the composite adaptive disturbance observer-based control for a class of nonlinear systems with multisource disturbance. Yang et al. $[24,25]$ have studied the consensus of second-order multiagent systems with exogenous disturbances and the robust consensus of multiagent systems with uncertain exogenous disturbances. Guo and Cao [26] have studied antidisturbance control for systems with multiple disturbances. Zhang et al. have studied the adaptive tracking of multiagent systems with stochastic communication noises [27, 28]. However, as far as we know, there are few researches done on the distributed coordination of fractional-order multiagent systems with exogenous disturbances.

In this paper, a class of fractional multiagent systems with external disturbances is studied. External disturbance signal is described with the dynamic subsystem, and expected reference information is interacted by a little part of agents. An adaptive pinning controller with a state observer is built to ensure multiple agents' states reaching the expected reference trajectory. Based on disturbance observers, the distributed coordination of fractional multiagent systems with external disturbances can be reached asymptotically by applying the controllers composted with the pinning controller.

\section{Problem Statements}

In this paper, distributed consensus of fractional multiagent system with external disturbance is investigated. Assume there are $n$ agents whose communication network is a directed graph. The multiagent systems are described as follows, for $i=1, \ldots, n$ :

$$
\begin{gathered}
x_{i}^{(\alpha)}(t)=\sum_{k \in N_{i}} a_{i k}\left(x_{k}(t)-x_{i}(t)\right)+u_{i}(t)+d_{i}(t) \\
y_{i}(t)=x_{i}(t),
\end{gathered}
$$

where $x_{i}(t), u_{i}(t)$, and $y_{i}(t)$ represent the state, control input, and system output of agent $i$, respectively, and $x_{i}(t) \in R^{m}$, $u_{i}(t) \in R^{m}$. In this paper, we assume $m=1$; for the cases of $m>1$, the similar conclusion can be got by applying Kronecker product. $x_{i}^{(\alpha)}(t)$ represents the $\alpha$-order derivative with $\alpha \in(0,1)$. The weight value $a_{i k}$ is the $(i, k)$ element of adjacency matrix $\mathrm{A}$, where $a_{i k}>0$ when agent $i$ can receive the information from agent $k$, otherwise $a_{i k}=0$. The set
$N_{i}=\left\{k: a_{i k}>0\right\}$ contains the neighbors of agent $i . d_{i}(t)$ is the external disturbance of the systems with the description:

$$
\begin{aligned}
& \xi_{i}^{(\alpha)}(t)=W \xi_{i}(t), \\
& d_{i}(t)=V \xi_{i}(t),
\end{aligned}
$$

where $W, V$ are the matrices of the system with $(W, V)$ observable.

Let $x(t)=\left[x_{1}(t), x_{2}(t), \ldots, x_{n}(t)\right]^{T}, u(t)=\left[u_{1}(t)\right.$, $\left.u_{2}(t), \ldots, u_{n}(t)\right]^{T}, d(t)=\left[d_{1}(t), d_{2}(t), \ldots, d_{n}(t)\right]^{T}$, then the cooperation protocol (1a) and (1b) can be rewritten as

$$
\begin{gathered}
x^{(\alpha)}(t)=-L x(t)+u(t)+d(t), \\
y(t)=x(t),
\end{gathered}
$$

where $y(t)=\left[y_{1}(t), y_{2}(t), \ldots, y_{n}(t)\right]^{T} . L$ is the Laplacian matrix satisfying with $L=D-A$, where $D=\operatorname{diag}\left\{D_{1}, D_{2}, \ldots\right.$, $\left.D_{n}\right\}$ and $D_{i}=\sum_{k=1}^{n} a_{i k}$. For any initial value, if the state of agent $i$ meets $\lim _{t \rightarrow \infty}\left(x_{i}(t)-x_{j}(t)\right)=0$, for all $i, j \in N$, the multiagent systems reach consensus asymptotically.

Assume that there is a reference leader (or a food source, an expected destination, etc.) in the multiagent systems, whose dynamics meets

$$
x_{0}^{(\alpha)}(t)=v_{0}(t)
$$

where $x_{0}(t)$ is the reference state expected agents moving tracking and $v_{0}(t)$ meets $v_{0}^{(\alpha)}(t)=u_{0}(t)$, while $u_{0}(t)$ is a continuous function satisfying the Lipschitz condition. The purpose of this paper is to design a distributed controller under the influence of external disturbance, for any initial value, agents of the system can track the reference tracking, or $\lim _{t \rightarrow \infty}\left(x_{i}(t)-x_{0}(t)\right)=0$, for all $i \in N$, then the multiagent systems reach consensus asymptotically and follow the reference trajectory.

Lemma 1 (see [8]). Assume that multiagent systems with $n$ agents exist a directed topology with a globally reachable node. Then matrix $L+B$ is full rank and the eigenvalues of matrix $L+B$ have positive real part, where $L$ is the Laplacian matrix of the connection topology of $n$ agents, and $B=\operatorname{diag}\left\{b_{i}, i=1, \ldots, n\right\}$, where $b_{i}$ is a nonnegative real number that does not always equal 0 .

Lemma 2 (see [29]). Assume that polynomial $q(s)=\rho_{0}+\rho_{1} s+$ $\cdots+\rho_{n} s^{n}$, and let $s=j \omega$ denote

$$
q(j \omega)=m(\omega)+j n(\omega) .
$$

And then $q(s)$ is Hurwitz stable if and only if the following conditions are satisfied with the following,

$$
\begin{aligned}
& \text { (1) The root of } m(\omega)=0, m_{1}<m_{2}<\cdots \text { and root of } \\
& n(\omega)=0, n_{1}<n_{2}<\cdots \text { meet } \\
& m_{1}<n_{1}<m_{2}<n_{2}<\cdots, \text { or } n_{1}<m_{1}<n_{2}<m_{2}<\cdots \text {. }
\end{aligned}
$$

(2) $m(0) n^{\prime}(0)-m^{\prime}(0) n(0)>0$. 
Lemma 3 (see [10]). Assume $A \in R^{n \times n}$ is set to be a deterministic real matrix, then the system

$$
\frac{d^{\alpha} x(t)}{d t^{\alpha}}=A x(t)
$$

can gradually reach stable under the following necessary and sufficient conditions:

$$
|\arg (\operatorname{spec}(A))|>\frac{\alpha \pi}{2}
$$

\section{Distributed Tracking for Fractional Multiagent Systems without Exogenous Disturbances}

Assume that the external disturbances of the system can be observed or can be ignored, we establish the state observer of the system (3a) and ( $3 b)$ :

$$
\begin{aligned}
& \widehat{x}^{(\alpha)}(t)=-L \widehat{x}(t)+u(t)+K_{1}(y(t)-\widehat{y}(t)), \\
& \widehat{y}(t)=\widehat{x}(t),
\end{aligned}
$$

where $K_{1}$ is the unknown parameters of the observer. $\hat{x}, \hat{y}$ are the estimated value of the state and output of the systems (3a) and (3b). Assume that the controller for agent $i$ is as follows:

$$
\begin{aligned}
u_{i}(t)= & v_{i}(t)-b_{i}\left(\widehat{x}_{i}(t)-x_{0}(t)\right), \\
v_{i}^{(\alpha)}(t)= & u_{0}(t) \\
& +c\left(\sum_{k \in N_{i}} a_{i k}\left(\widehat{x}_{k}(t)-\widehat{x}_{i}(t)\right)+b_{i}\left(x_{0}(t)-\widehat{x}_{i}(t)\right)\right),
\end{aligned}
$$

where $b_{i} \geq 0$, if $b_{i}>0$ represents agent $i$ can sense the reference status, otherwise it cannot sense the perceived expectations. The control gain $c>0$ is an undetermined parameter.

Let $\delta(t)=x(t)-x_{0}(t), e_{x}(t)=x(t)-\widehat{x}(t), e_{v}(t)=v(t)-$ $v_{0}(t)$, and $v(t)=\left[v_{1}(t), \ldots, v_{n}(t)\right]^{T}$, according $(3 \mathrm{a})$ and $(3 \mathrm{~b})$, (4), (9), and (10), the following equations can be got:

$$
\begin{aligned}
& \delta^{(\alpha)}(t)=-(L+B) \delta(t)+e_{v}(t)+B e_{x}(t), \\
& e_{v}^{(\alpha)}(t)=-c(L+B) \delta(t)+c(L+B) e_{x}(t), \\
& e_{x}^{(\alpha)}(t)=-\left(L+K_{1}\right) e_{x}(t) .
\end{aligned}
$$

Theorem 4. Assume that the connection topology of multiagent systems is directed network with $n$ agents, and there exists a globally reachable node. There are lots of agents including the globally reachable node can sense the information from the reference node. Then the distributed cooperation of multiagent systems (3a) and (3b) without external disturbance, by applying the state observer (9) and the controller (10), can be obtained asymptotically if

(1) $K_{1}=\operatorname{diag}\left\{k_{1 i}, i \in I\right\}$, where $k_{1 i}$ are nonnegative real numbers that are not all zero;

(2) the control gain $c>0$, and $c<\min \left\{\left|\mu_{i}\right|^{2} /\right.$ $\left.\operatorname{Re}\left(\mu_{i}\right)\left|\operatorname{Im}\left(\mu_{i}\right)\right|^{2}, i \in I\right\}$, where $\mu_{i}$ is the eigenvalue for the matrix $\bar{L}=L+B$ and $\mu_{i}=\operatorname{Re}\left(\mu_{i}\right)+j \operatorname{Im}\left(\mu_{i}\right)$.

Proof. Let $\vartheta_{1}(t)=\left[\delta(t), e_{v}(t), e_{x}(t)\right]^{T}$, according to the system (11a), (11b), and (11c)

$$
\vartheta_{1}^{(\alpha)}(t)=\Psi_{1} \vartheta_{1}(t)
$$

where $\Psi_{1}=\left[\begin{array}{ccc}-\bar{L} & I & B \\ -c \bar{L} & 0 & c \bar{L} \\ 0 & 0 & -\left(L+K_{1}\right)\end{array}\right], \bar{L}=L+B$. The characteristic equation of system (12) can be got according to the Laplace transform.

$$
\operatorname{det}\left(s^{\alpha}-\Psi_{1}\right)=0 .
$$

Let $\lambda=s^{\alpha}$, then the above equation is equivalent to

$$
\operatorname{det}\left(\lambda-\Psi_{1}\right)=0,
$$

that is,

$$
\begin{gathered}
\operatorname{det}(\lambda(\lambda+\bar{L})+c \bar{L})=0, \\
\operatorname{det}\left(\lambda+\left(L+K_{1}\right)\right)=0 .
\end{gathered}
$$

According to Lemma 1, the matrix $L+K_{1}$ is of full rank and its eigenvalues are all with positive real part. So the eigenvalues of characteristic equation (15b) have negative real parts. The eigenvalues of characteristic equation (15b) $s=\lambda^{1 / \alpha}$ are satisfied with the condition in Lemma 3.

Similarly, if $B=\operatorname{diag}\left\{b_{i}, i \in I\right\}$, where $b_{i}$ are nonnegative real numbers which are not all zeros. Assume the eigenvalues of matrix $\bar{L}$ are $\mu_{i}=a+j b$, where $a>0$, and $j$ is the plural unit. According to the characteristic equation (15a)

$$
\lambda^{2}+\lambda \mu_{i}+c \mu_{i}=0
$$

Let $q(s)=\lambda^{2}+\lambda(a+j b)+c(a+j b)$, the following proof is to prove the eigenvalues of (16) are all in the left half-plane of the complex space. Let $\lambda=j \omega$, then (16) becomes

$$
q(\omega)=m(\omega)+j n(\omega),
$$

where $m(\omega)=-\omega^{2}+c a-b \omega, n(\omega)=c b+a \omega$. Due to $m(0)=c a$, $m^{\prime}(0)=-b, n(0)=c b$, and $n^{\prime}(0)=a$; there is $m(0) n^{\prime}(0)-$ $m^{\prime}(0) n(0)=c\left(a^{2}+b^{2}\right)=c\left|\mu_{i}\right|^{2}>0$. The roots of equation $m(\omega)=0$ and $n(\omega)=0$ are

$$
m_{1,2}=\frac{-b \pm \sqrt{b^{2}+4 c a}}{2}, \quad n=-\frac{c b}{a} .
$$

Let $\left(-b-\sqrt{b^{2}+4 c a}\right) / 2<-c b / a<\left(-b+\sqrt{b^{2}+4 c a}\right) / 2$, There is

$$
c<\frac{\left|\mu_{i}\right|^{2}}{a|b|^{2}}
$$


Therefore, according to Lemma 2, when the control gain of the multiagent system meets the conditions of Theorem 4, the eigenvalues of (16) are all in the left half-plane of the complex space. By means of $s=\lambda^{1 / \alpha}$, the condition in Lemma 3 is satisfied, and the systems are stable. The system (12) will be converged on the equilibrium point. That is, for all agents $i, \lim _{t \rightarrow \infty} \delta_{i}(t)=0$, there is $\lim _{t \rightarrow+\infty} x_{i}(t)-x_{0}(t)=0$. Theorem 4 is proved.

Corollary 5. Assume that the connection topology of multiagent systems is directed and symmetrical network with $n$ agents and an interacted reference node. Then the distributed cooperation of multiagent systems (3a) and (3b) without external disturbance, by applying the state observer (9) and the controller (10), can be reached asymptotically, if

(1) $K_{1}=\operatorname{diag}\left\{k_{1 i}, i \in I\right\}$, where $k_{1 i}$ is a nonnegative real number which is not all zero;

(2) control gain of the controller $c>0$.

\section{Distributed Tracking for Fractional Multiagent Systems with External Disturbance}

Assume that the systems are influenced by external disturbances, the dynamical equation with disturbances can be rewritten as

$$
\begin{aligned}
z^{(\alpha)}(t) & =A_{0} z(t)+H_{1} u(t), \\
y_{z}(t) & =H_{2} z(t),
\end{aligned}
$$

where $z(t)=[x(t), \xi(t)]^{T}, y_{z}(t)=[y(t), d(t)]^{T}, A_{0}=$ $\left[\begin{array}{cc}-L & V \\ 0 & W\end{array}\right]$, and $H_{1}=\left[\begin{array}{l}I \\ 0\end{array}\right], H_{2}=\left[\begin{array}{ll}I & 0 \\ 0 & V\end{array}\right]$. Based on the system equations with external disturbances, the following observer is established:

$$
\begin{gathered}
\widehat{z}^{(\alpha)}(t)=A_{0} \widehat{z}(t)+H_{1} u(t)+K\left(y_{z}(t)-\widehat{y}_{z}(t)\right), \\
\widehat{y}_{z}(t)=H_{2} \widehat{z}(t),
\end{gathered}
$$

where $\widehat{z}(t)=[\widehat{x}(t), \widehat{\xi}(t)]^{T}, \widehat{y}_{z}(t)=[\widehat{y}(t), \widehat{d}(t)]^{T}, K=$ $\left[\begin{array}{ll}K_{1} & \\ & K_{2}\end{array}\right]$ is the matrix to be determined. Let $e(t)=z(t)-$ $\widehat{z}(t)=\left[\begin{array}{c}e_{x}(t) \\ e_{\xi}(t)\end{array}\right]=\left(\begin{array}{c}x(t)-\widehat{x}(t) \\ \xi(t)-\widehat{\xi}(t)\end{array}\right)$, there is

$$
e^{(\alpha)}(t)=\bar{A}_{0} e(t),
$$

where $\bar{A}_{0}=A_{0}-K H_{2}$.

Theorem 6. Assume that the connection topology of multiagent systems is directed network with $n$ agents, and there exists a globally reachable node. A lot of agents including the globally reachable node can sense the information from the reference node. Then the distributed cooperation of multiagent systems (3a) and (3b) with external disturbance, by applying the state observer (21), can be obtained asymptotically, if

(1) $K_{1}=\operatorname{diag}\left\{k_{1 i}, i \in I\right\}$, where $k_{1 i}$ are nonnegative real numbers which are not all zeros;

(2) $W-K_{2} V$ is negative definite.
Proof. Assume that the controller for multiagent system is

$$
\begin{aligned}
u_{i}(t)= & v_{i}(t)-b_{i}\left(\widehat{x}_{i}(t)-x_{0}(t)\right)-\widehat{d}_{i}(t), \\
v_{i}^{(\alpha)}(t)= & u_{0}(t) \\
& +c\left(\sum_{k \in N_{i}} a_{i k}\left(\widehat{x}_{k}(t)-\widehat{x}_{i}(t)\right)+b_{i}\left(x_{0}(t)-\widehat{x}_{i}(t)\right)\right) .
\end{aligned}
$$

This adaptive pinning controller is proposed on the base of the controller (10), where observed disturbances $\widehat{d}_{i}(t)=$ $V \widehat{\xi}_{i}(t)$. So that the original systems (3a) and (3b) are described as:

$$
x^{(\alpha)}(t)=-L x(t)+v(t)-B(\widehat{x}(t)-\bar{x})+d(t)-\widehat{d}(t),
$$

where $v(t)=\left[v_{1}(t), \ldots, v_{n}(t)\right]^{T}, B=\operatorname{diag}\left\{b_{i}, i=1, \ldots, n\right\}$. Let $\delta(t)=x(t)-x_{0}(t)$, then there is:

$$
\delta^{(\alpha)}(t)=-(L+B) \delta(t)+v(t)-v_{0}(t)+B e_{x}(t)+V e_{\xi}(t) .
$$

Let $e_{v}(t)=v(t)-v_{0}(t)$, the state of the controller (23) becomes

$$
e_{v}^{(\alpha)}(t)=c\left(L e_{x}(t)-L \delta(t)-B \delta(t)+B e_{x}(t)\right)
$$

The closed-loop system can be got according to (25), (26), and (22):

$$
\begin{aligned}
& \delta^{(\alpha)}(t)=-(L+B) \delta(t)+v(t)-v_{0}(t)+B e_{x}(t)+V e_{\xi}(t), \\
& e_{v}^{(\alpha)}(t)=-c(L+B) \delta(t)+c(L+B) e_{x}(t), \\
& e^{(\alpha)}(t)=\bar{A}_{0} e(t),
\end{aligned}
$$

where $\bar{A}_{0}=A_{0}-K H_{2}, e(t)=\left[e_{x}(t), e_{\xi}(t)\right]^{T}$. Let $\vartheta(t)=$ $\left[\delta(t), e_{v}(t), e_{x}(t), e_{\xi}(t)\right]^{T}$, then there are

$$
\vartheta^{(\alpha)}(t)=\Psi \vartheta(t)
$$

where

$$
\Psi=\left[\begin{array}{cccc}
-\bar{L} & I & B & V \\
-c \bar{L} & 0 & c \bar{L} & 0 \\
0 & 0 & -\left(L+K_{1}\right) & V \\
0 & 0 & 0 & W-K_{2} V
\end{array}\right], \quad \bar{L}=L+B
$$

Applying the proof procedure in Theorem 4, we can get the condition (1) in Theorem 6 . Based on the $(W, V)$ observable, the condition (2) in Theorem 6 can be ensured. 


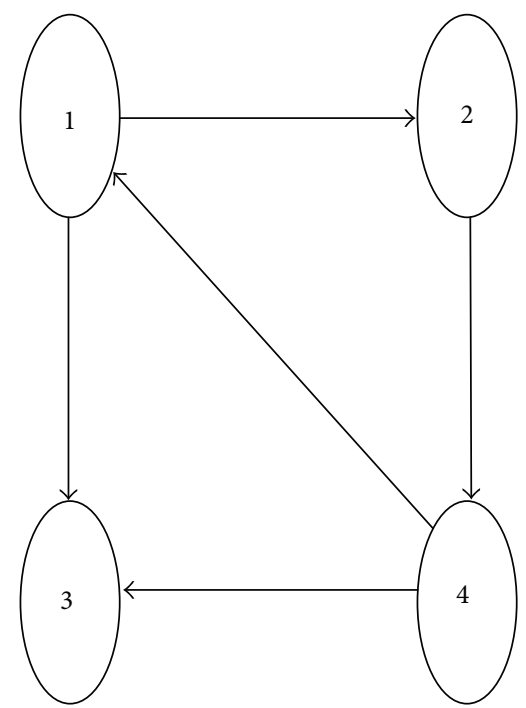

FIgURE 1: Topology graph of multiagent systems.

\section{Examples}

Consider undirected connected network topologies (Figure 1) where only agent1 is connected with a reference leader. The connected weighted values of the adjacency matrix are generated randomly in the range $[0,2]$. The order of fractional dynamical multiagent systems is $\alpha=0.8$.

Case 1. Movement trajectory of Multiagent systems without disturbances will be studied. We select the initial states of agents randomly in the area $[0,10]$, the reference trajectory is $x_{0}=0.15$, and the control gains $b_{1}=1, c=1$. The moving tracking of agent systems without disturbances are plotted in Figure 2 with $k_{11}=1$, where the distributed cooperation is reached asymptotically.

Case 2. Movement trajectory of Multiagent systems with disturbances will be studied. Suppose the exogenous disturbance system (2) is set as

$$
W=\left(\begin{array}{cc}
0 & 2 \\
-2 & 0
\end{array}\right), \quad V=\left(\begin{array}{ll}
0 & 1
\end{array}\right)
$$

with initialized value $\xi_{i}(0)=[0.5 \sin 1,0.5 \cos 1]^{T}$. The output of the fractional-order systems with disturbances is shown in Figure 3. By using linear matrix inequalities packages of MATLAB, we get $K_{2}=(4 / 5)[4,3]^{T}$. The moving tracking of fractional systems with exogenous disturbances is plotted in Figure 4, where the expected reference tracking has been achieved for multiagent systems with the disturbance observers.

\section{Conclusions}

In this paper, distributed coordination of fractional multiagent systems with extrinsic disturbances is studied. A state observer of the dynamical system is developed based on

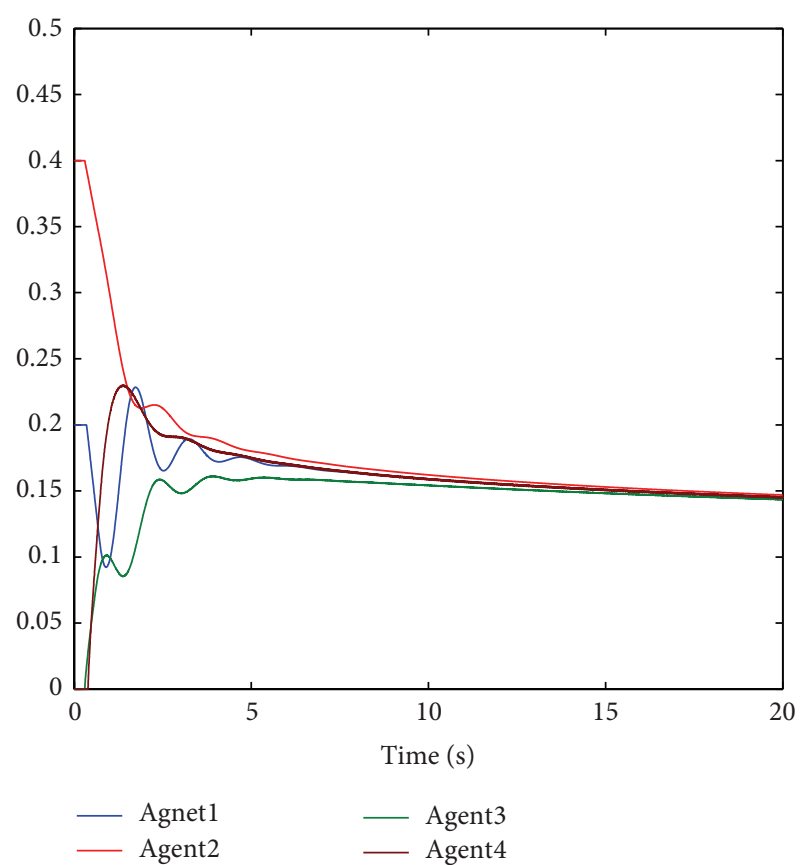

FIGURE 2: Movement trajectory of multiagent systems without disturbance.

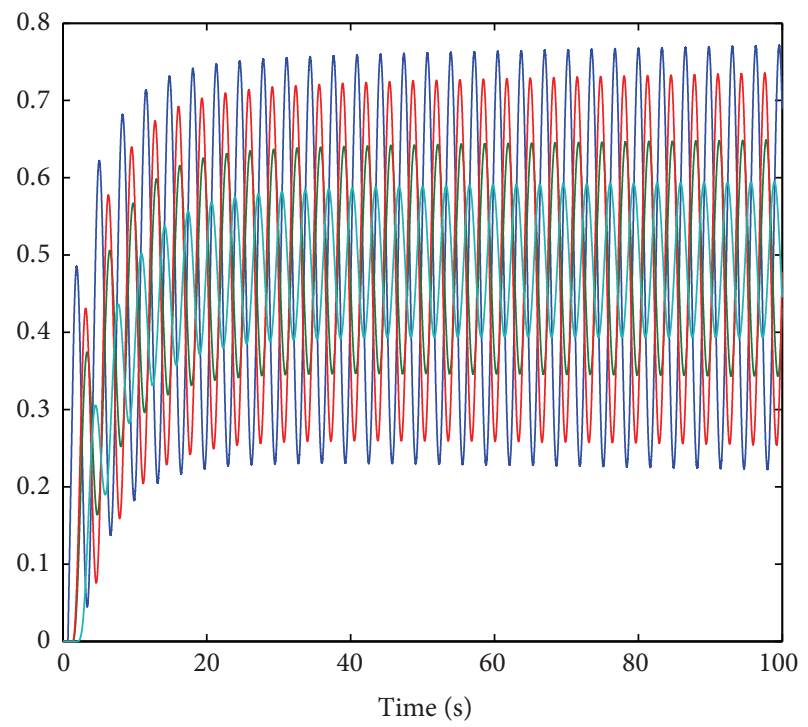

FIGURE 3: Movement trajectory of multiagent systems with disturbance.

the disturbance observers; an adaptive pinning controller is presented based on the estimated disturbances information from the disturbance observers. With the reference tracking interacted, a fractional composite controller for the antiinterference of multiagent system is proposed, which can make the multiagent systems with external disturbance reach the reference trajectory asymptotically. 


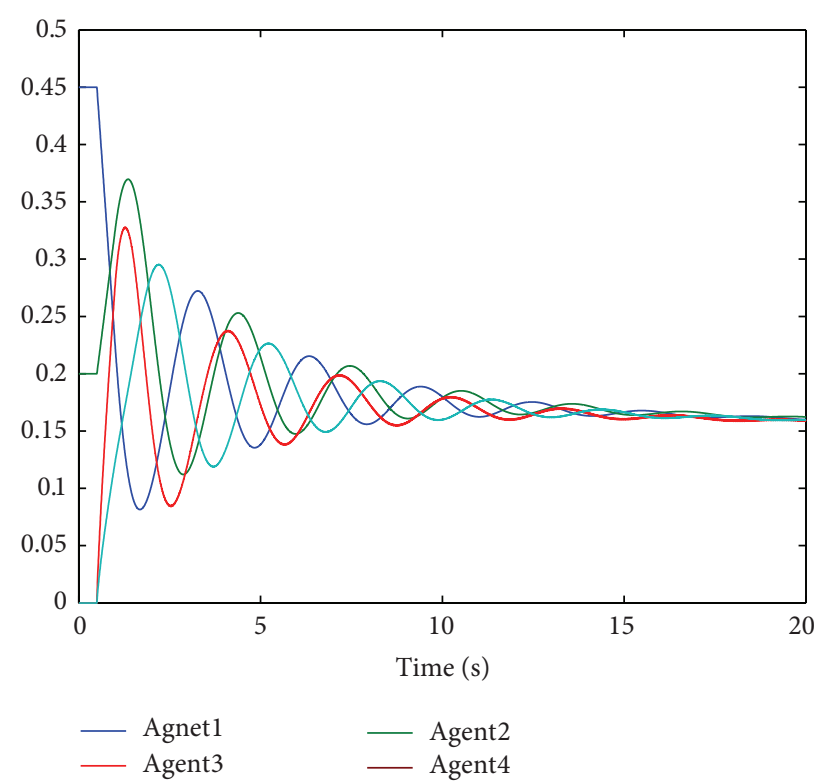

FIGURE 4: Moving trajectory of multiagent systems with disturbance observers.

\section{Conflict of Interests}

The authors declare that there is no conflict of interests regarding the publication of this paper.

\section{Acknowledgments}

This research is supported in part by the State Key Development Program for Basic Research of China (Grant no. 2012CB720003), the National Natural Science Foundation of China (Grant nos. 61273152, 61127007, 61170161, 61174085, and 61304052), the Natural Science Foundation of Shandong Province of China (no. ZR2011FM017), the Science Foundation of China postdoctoral (under Grant 2011M500215), and the Outstanding Young Research Award Fund of Shang Province of China (under Grant BS2013DX005).

\section{References}

[1] R. Olfati-Saber, J. A. Fax, and R. M. Murray, "Consensus and cooperation in networked multi-agent systems," Proceedings of the IEEE, vol. 95, no. 1, pp. 215-233, 2007.

[2] W. Ren, R. W. Beard, and E. M. Atkins, "Information consensus in multivehicle cooperative control: collective group behavior through local interaction," IEEE Control Systems Magazine, vol. 27, no. 2, pp. 71-82, 2007.

[3] S. Li, H. Du, and X. Lin, "Finite-time consensus algorithm for multi-agent systems with double-integrator dynamics," Automatica, vol. 47, no. 8, pp. 1706-1712, 2011.

[4] H. Y. Yang, X. L. Zhu, and S. Y. Zhang, "Consensus of secondorder delayed multi-agent systems with leader-following," European Journal of Control, vol. 16, no. 2, pp. 188-199, 2010.

[5] W. Ren and R. W. Beard, "Consensus seeking in multiagent systems under dynamically changing interaction topologies,"
IEEE Transactions on Automatic Control, vol. 50, no. 5, pp. 655661, 2005.

[6] L. Moreau, "Stability of multiagent systems with time-dependent communication links," IEEE Transactions on Automatic Control, vol. 50, no. 2, pp. 169-182, 2005.

[7] W. Ren, "On consensus algorithms for double-integrator dynamics," IEEE Transactions on Automatic Control, vol. 53, no. 6, pp. 1503-1509, 2008.

[8] W. Ren and R. W. Beard, Distributed Consensus in MultiVehicle Cooperative Control: Theory and Applications, Springer, London, UK, 2008.

[9] S. Das, Functional Fractional Calculus, Springer, New Delhi, India, 2011.

[10] I. Podlubny, Fractional Differential Equations, Academic Press, San Diego, Calif, USA, 1999.

[11] W. Ren and Y. C. Cao, Distributed Coordination of Multi-Agent Networks, Springer, London, UK, 2011.

[12] Y. C. Cao, Y. Li, W. Ren, and Y. Q. Chen, "Distributed coordination of networked fractional-order systems," IEEE Transactions on Systems, Man, and Cybernetics B: Cybernetics, vol. 40, no. 2, pp. 362-370, 2010.

[13] T. Kaczorek, "Fractional positive continuous-time linear systems and their reachability," International Journal of Applied Mathematics and Computer Science, vol. 18, no. 2, pp. 223-228, 2008.

[14] D. Qian, C. Li, R. P. Agarwal, and P. J. Y. Wong, "Stability analysis of fractional differential system with Riemann-Liouville derivative," Mathematical and Computer Modelling, vol. 52, no. 5-6, pp. 862-874, 2010.

[15] A. Atangana and A. Kilicman, "Analytical solutions of the spacetime fractional derivative of advection dispersion equation," Mathematical Problems in Engineering, vol. 2013, Article ID 853127, 9 pages, 2013.

[16] A. Atangana and A. Kilicman, "A novel integral operator transform and its application to some FODE and FPDE with some kind of singularities," Mathematical Problems in Engineering, vol. 2013, Article ID 531984, 7 pages, 2013.

[17] A. Atangana, N. Bildik, and S. C. O. Noutchie, "New iteration methods for time-fractional modified nonlinear Kawahara equation," Abstract and Applied Analysis, vol. 2014, Article ID 740248, 9 pages, 2014

[18] Y. Cao and W. Ren, "Distributed formation control for fractional-order systems: dynamic interaction and absolute/relative damping," Systems and Control Letters, vol. 59, no. 3-4, pp. 233240, 2010.

[19] H. Y. Yang, L. Guo, B. Xu, and J. Gu, "Collaboration control of fractional-order multiagent systems with sampling delay," Mathematical Problems in Engineering, vol. 2013, Article ID 854960, 7 pages, 2013.

[20] H. Yang, L. Guo, X. Zhu, K. Cao, and H. Zou, "Consensus of compound-order multi-agent systems with communication delays," Central European Journal of Physics, vol. 11, no. 6, pp. 806-812, 2013.

[21] H. Y. Yang, X. Zhu, and K. Cao, "Distributed coordination of fractional order multi-agent systems with communication delays," Fractional Calculus and Applied Analysis, vol. 17, no. 1, pp. 23-37, 2014.

[22] L. Guo and W.-H. Chen, "Disturbance attenuation and rejection for systems with nonlinearity via DOBC approach," International Journal of Robust and Nonlinear Control, vol. 15, no. 3, pp. 109-125, 2005. 
[23] X. Wei, N. Chen, and W. Li, "Composite adaptive disturbance observer-based control for a class of nonlinear systems with multisource disturbance," International Journal of Adaptive Control and Signal Processing, vol. 27, no. 3, pp. 199-208, 2013.

[24] H. Y. Yang, Z. X. Zhang, and S. Y. Zhang, "Consensus of secondorder multi-agent systems with exogenous disturbances," International Journal of Robust and Nonlinear Control, vol. 21, no. 9, pp. 945-956, 2011.

[25] H. Yang, L. Guo, and C. Han, "Robust consensus of multi-agent systems with uncertain exogenous disturbances," Communications in Theoretical Physics, vol. 56, no. 6, pp. 1161-1166, 2011.

[26] L. Guo and S. Cao, Anti-Disturbance Control for Systems with Multiple Disturbances, CRC Press, New York, NY, USA, 2013.

[27] Q. Zhang and J. F. Zhang, "Adaptive tracking games for coupled stochastic linear multi-agent systems: stability, optimality and robustness," IEEE Transactions on Automatic Control, vol. 58, no. 11, pp. 2862-2877, 2013.

[28] T. Li and J. Zhang, "Consensus conditions of multi-agent systems with time-varying topologies and stochastic communication noises," IEEE Transactions on Automatic Control, vol. 55, no. 9, pp. 2043-2057, 2010.

[29] K. Ogata, Discrete-Time Control System, Prentice-Hall, Englewood Cliffs, NJ, USA, 1995. 


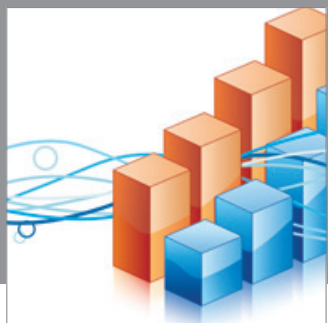

Advances in

Operations Research

mansans

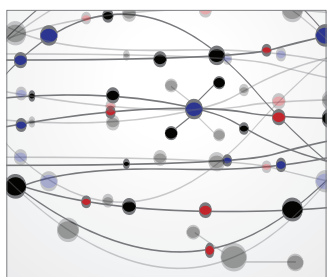

The Scientific World Journal
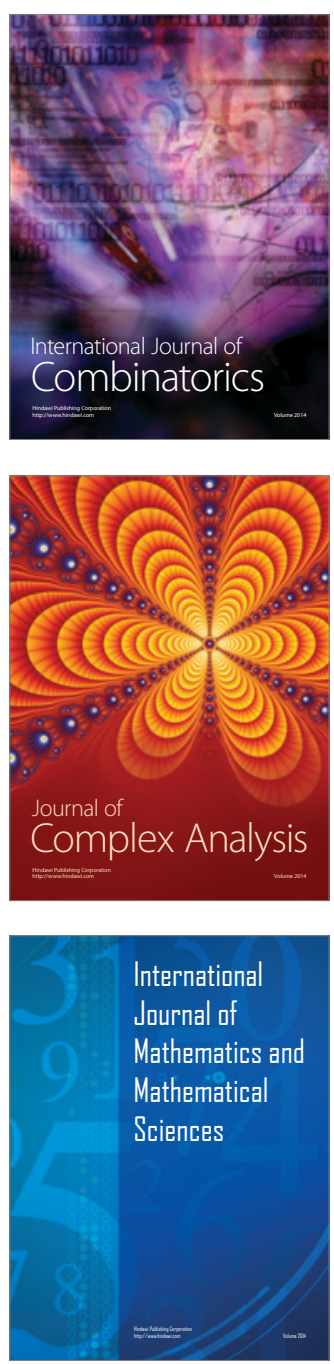
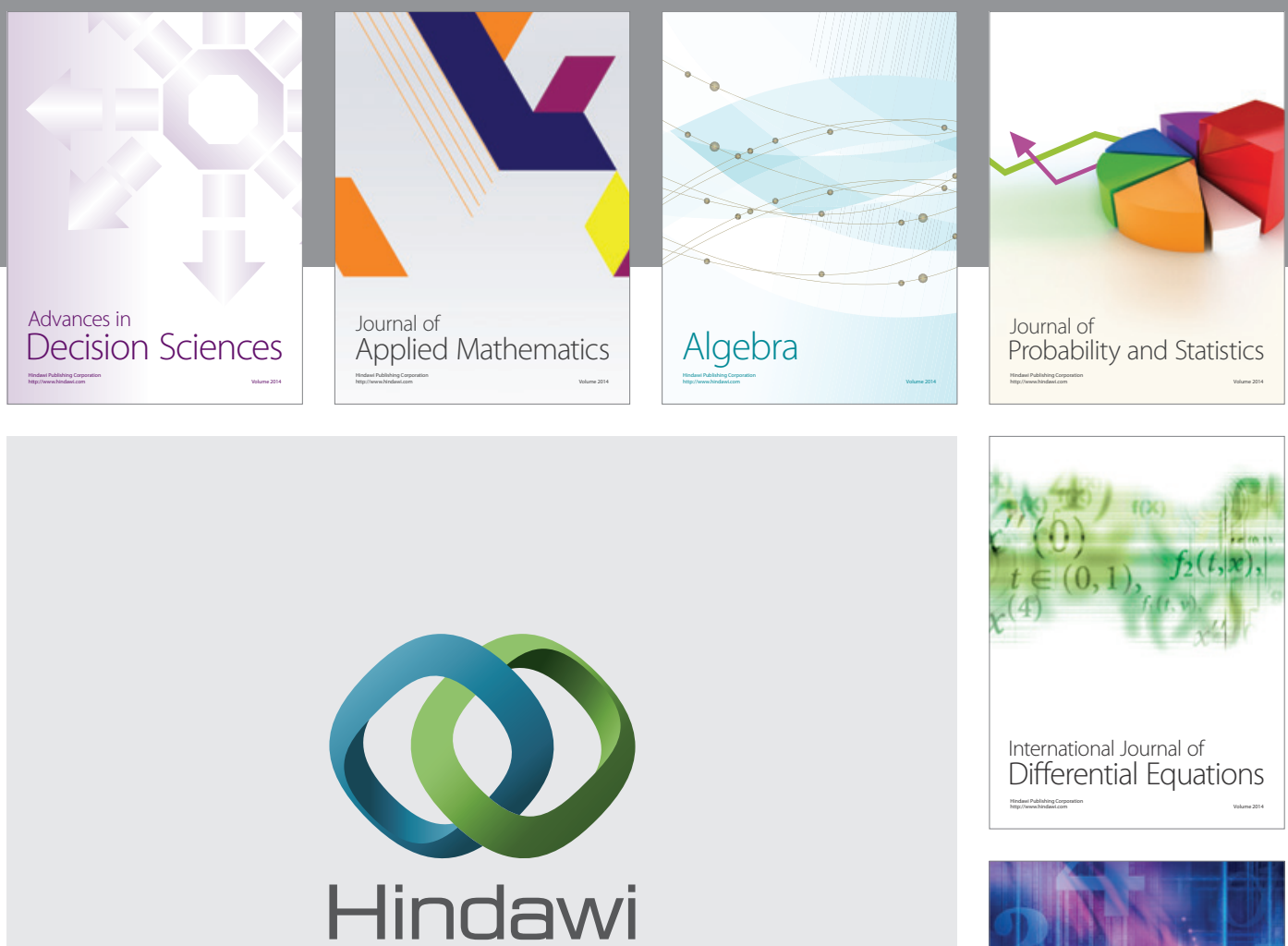

Submit your manuscripts at http://www.hindawi.com
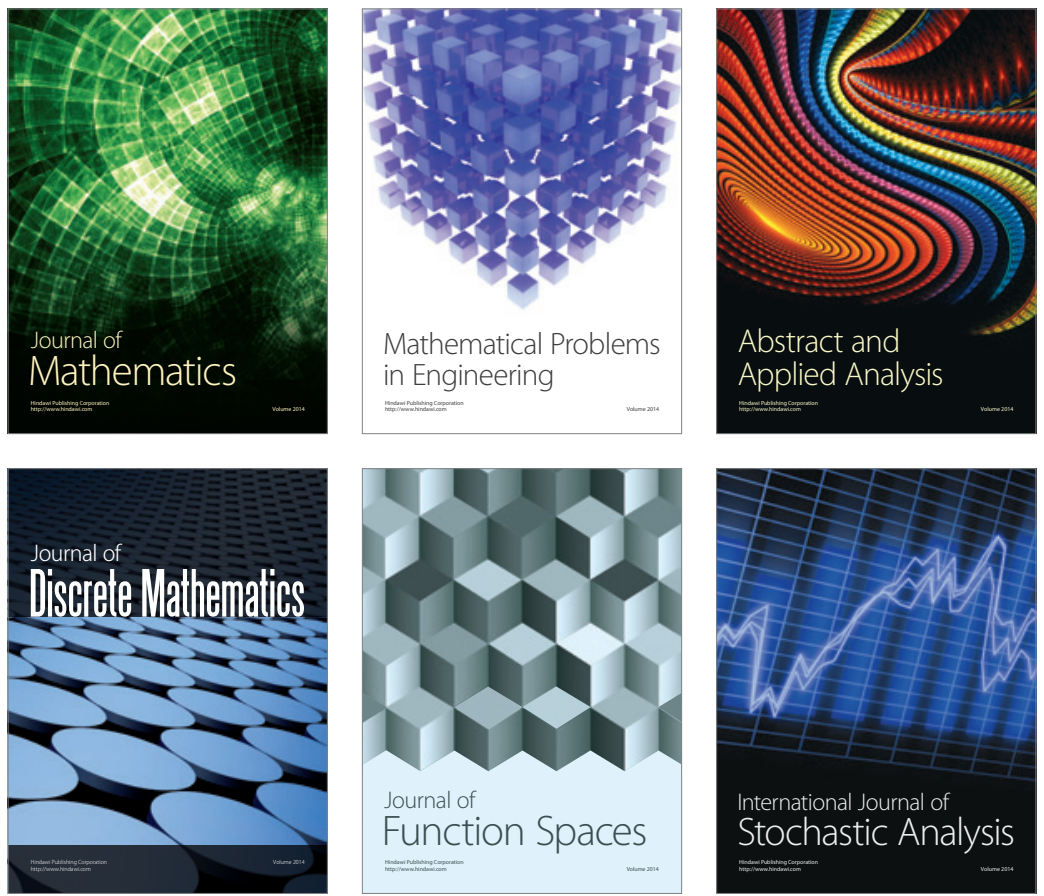

Journal of

Function Spaces

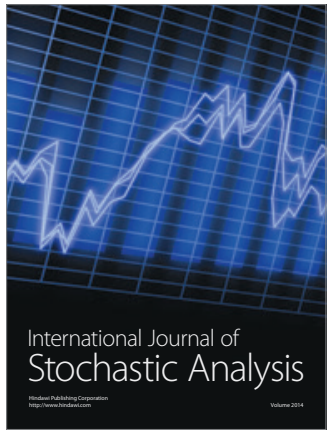

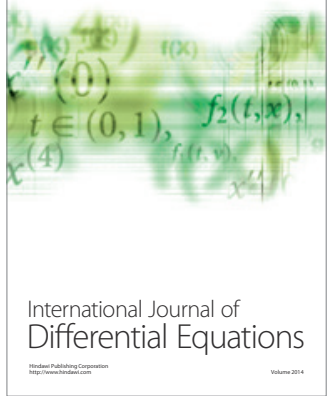
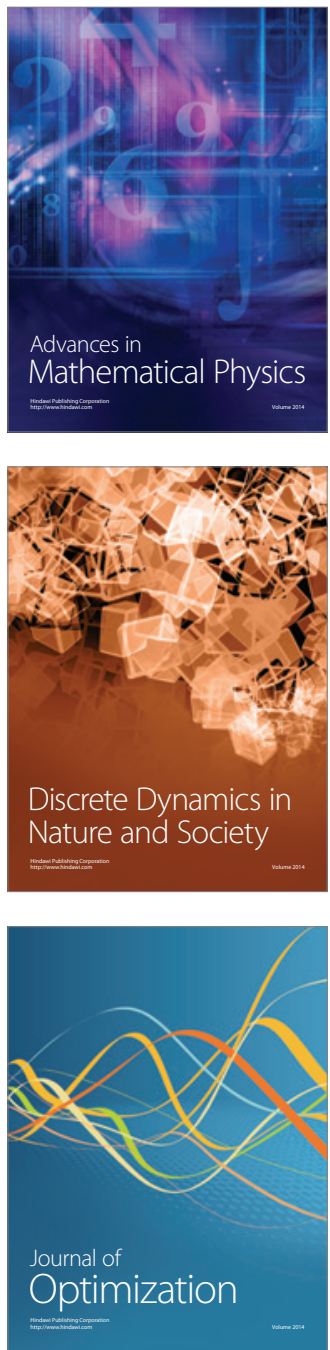